Chirurg 2012 $\cdot 83: 384$

DOI 10.1007/s00104-012-2289-7

Online publiziert: 15. März 2012

(c) Springer-Verlag 2012

\title{
K. Lorenz
}

Medizinische Fakultät, Klinik für Allgemein-, Viszeral- und Gefäßchirurgie,

Universitätsklinikum Halle, Halle an der Saale

\section{Kalzimimetika: Keine Konkurrenz zur Operation beim primären Hyperparathyreoidismus}

nisch-morphologische Erscheinungsform, intraoperative PTH-Schnellbestimmung und frühpostoperative Hypokalzämierate bei 281 sporadischen pHPT-Operationen. Nach positiver Lokalisationsdiagnostik (Ultraschall, $\mathrm{Tc}^{99 \mathrm{~m}}$-Sestamibi -Szintigraphie) erfolgte eine fokussierte Operation, bei negativer eine bilaterale Exploration jeweils mit intraoperativer PTH-Schnellbestimmung. 118 Patienten nahmen zum Operationszeitpunkt Kalziumsenker. Diese beeinflussten die Lokalisationsdiagnostik nicht, die Mehrdrüsenhyperplasierate war nicht erhöht. Höhere präoperative Serumkalziumkonzentrationen unter Kalziumsenkern wiesen auf eine Eindrüsenerkrankung hin.

Unter Kalziumsenkern zeigte die intraoperative PTH-Dynamik einen steileren Abfall und geringeren PTH-Wiederanstieg, ausgeprägt bei Kalzimimetika und Bisphosphonaten, ohne Erfordernis zusätzlicher Bestimmungen. Kombinierte Kalziumsenker zeigten frühpostoperativ signifikant häufiger eine Hypokalziämie (OR: 3,06; 95\%CI: 1,21-7,75), insbesondere die Kombination Kalzimimetika/ Bisphosphonate.

Die Studie zeigt trotz Schwächen (retrospektiv, geringe Fallzahl) einen Trend konservativer Therapieversuche bei operationspflichtigem pHPT: Der Verbreitungsgrad kalziumsenkender Medikation, hier $42 \%$ für Patienten mit pHPT, die schließlich operiert werden, ist hoch und außerhalb der Indikation dieser Substanzgruppen. Die Kosten, Nebenwirkungen und der ausbleibende dauerhafte Erfolg der Kalziumsenker machen deutlich, dass die Operation unverändert die The- rapie der Wahl beim pHPT darstellt und am schnellsten zur dauerhaften Heilung führt. Die erhöhte postoperative Hypokalzämierate bei präoperativer Kombinationsgabe von Kalziumsenkern ist ein weiteres Argument, die Anwendung dieser Medikamente für die Indikation des pHPT infrage zu stellen.

\section{Korrespondenzadresse}

PD Dr. K. Lorenz

Medizinische Fakultät, Klinik für Allgemein-, Viszeral- und Gefäßchirurgie, Universitätsklinikum Halle, Ernst-Grube-Str. 40, 06120 Halle an der Saale kerstin.lorenz@uk-halle.de

Interessenkonflikt. Die korrespondierende Autorin gibt an, dass kein Interessenkonflikt besteht.

\section{Literatur}

1. Wermers RA, Khosla S, Atkinson EJ et al (2006) Incidence in primary hyperparathyroidism in Rochester, Minnesota, 1993-2001: an update on the changing epidemiology of the disease. J Bone Miner Res 21:171-177

2. NIH Conference (1991) Diagnosis and management of asymptomatic primary hyperparathyroidism: consensus development conference statement. Ann Intern Med 114:593-597

3. Bilezekian JP, Khan AA, Potts JT (2009) Guidelines for the management of asymptomatic primary hyperparathyroidism: summary statement from the third international workshop. J Clin Endocrinol Metab 94:335-339

4. Dillon ML, Frazee LA (2011) Cincalcet for the treatment of primary hyperparathyroidism. Am JTher 18:313-322

5. Bollerslev J, Marcocci C, Sosa M et al (2011) Current evidence for recommendation of surgery, medical treatment and vitamin D repletion in mild primar hyperparathyroidism. Eur J Endocrinol 1265:851864 